\title{
Meteor44 Video Meteor Photometry
}

\author{
Wesley R. Swift ${ }^{1}$, Robert M. Suggs ${ }^{2}$, and William J. Cooke ${ }^{2}$
}

\begin{abstract}
Meteor 44 is a software system developed at MSFC for the calibration and analysis of video meteor data. The dynamic range of the (8bit) video data is extended by approximately 4 magnitudes for both meteors and stellar images using saturation compensation. Camera and lens specific saturation compensation coefficients are derived from artificial variable star laboratory measurements. Saturation compensation significantly increases the number of meteors with measured intensity and improves the estimation of meteoroid mass distribution. Astrometry is automated to determine each image's plate coefficient using appropriate star catalogs. The images are simultaneously intensity calibrated from the contained stars to determine the photon sensitivity and the saturation level referenced above the atmosphere. The camera's spectral response is used to compensate for stellar color index and typical meteor spectra in order to report meteor light curves in traditional visual magnitude units. Recent efforts include improved camera calibration procedures, long focal length "streak" meteor photometry and two-station track determination. Meteor 44 has been used to analyze data from the 2001, 2002 and 2003 MSFC Leonid observational campaigns as well as several lesser showers. The software is interactive and can be demonstrated using data from recent Leonid campaigns.
\end{abstract}

1) Raytheon / MSFC Group, Space Environments Team, EV13, Marshall Space Flight Center, Huntsville, AL 35812

2) NASA, Space Environments Team, EV13, Marshall Space Flight Center, Huntsville, AL 35812 


\title{
Meteor44 Video Meteor Photometry
}

\author{
Wesley R. Swift, Robert M. Suggs, and William J. Cooke
}

\subsection{Introduction}

The motivation is to minimize the risk to existing and proposed space assets by the estimation of space environment conditions and effects. Meteor storms are a significant risk to operational satellites. Since mitigation measures imply down time, accurate prediction saves operators money. The goal for video meteor photometry is to acquire and analyze video meteor observations with the intent to determine rates and population indices, which can then be used to constrain the stream models that form the basis of activity forecasts. The demand for such forecasts is significant: Over $\mathbf{4 0}$ satellites requested predictions for the 2001 Leonid meteor storm.

Accurate photometry is needed to determine mass flux spectrum and thus spacecraft hazard. In the past, the MeteorScan [Gural, 1995] program for intensified video meteor analysis has been very good for meteor detections but weak on photometry. Saturation of the meteor images introduces serious non-linearity that has in the past limited the video photometry dynamic range: most bright meteors are saturated. Lens falloff, sky background, background variations and sky transparency are also calibration concerns. Furthermore, comparisons between visual and second and third generation (GENII and GENIII) intensified observations are difficult at best due to the lack of instrument spectral response compensation. To overcome these obstacles, "Meteor44" was written to make use of on-sky flat-fields, stellar intensity and astrometry calibrations, meteor spectra, and camera / lens specific saturation compensation to improve meteor photometry.

\subsection{Meteor44 System Overview}

Meteor44 was initially conceived as photometric program to follow MeteorScan. Although direct to disk data gathering is possible, the meteor video is usually recorded on tape as shown in Figure 1a and only later converted to Microsoft Audio Video Interface (AVI) files for processing by MeteorScan and Meteor44. The edited output $\log$ (LGE) from MeteorScan and the AVI files are the input to Meteor44.

As shown in Figure 1b, Meteor44 is comprised of three main components: 1) "Condense" drastically reduces the size of the video data by removing frames with no meteor activity while retaining the auxiliary data, 2) "Photometry" produces calibrated meteor light curves with associated astrometric data, and 3) "VideoCal" provides instrument specific saturation compensation. These components are supplemented with numerous utilities for viewing, plotting, sorting, browsing, and quality controlling the video and meteor data. The software is written in IDL, the Interactive Data Language by Research Systems, Inc. By structuring the program as an interactive graphical use interface (GUI), one is able to connect multiple data handling, analysis and visualization tools in a powerful, intuitive format. The lack of native DL video read procedures required the development of an external $\mathrm{C}_{++}$ based dynamic link library module (avi.dll) to read AVI files. Simple IDL procedures call the avi.dll in order to read and write individual frames of the video 
data. The shareware frame server AviSynth from sourceforge.net is used to handle the many mutant digital formats. The combination works extremely well together and allows one to play the video or view and process individual frames from within IDL without requiring vast computer resources. The program is called "Meteor44" to reflect its origin within the ED44 group of the Engineering Directorate of NASA's Marshal Space Flight Center.

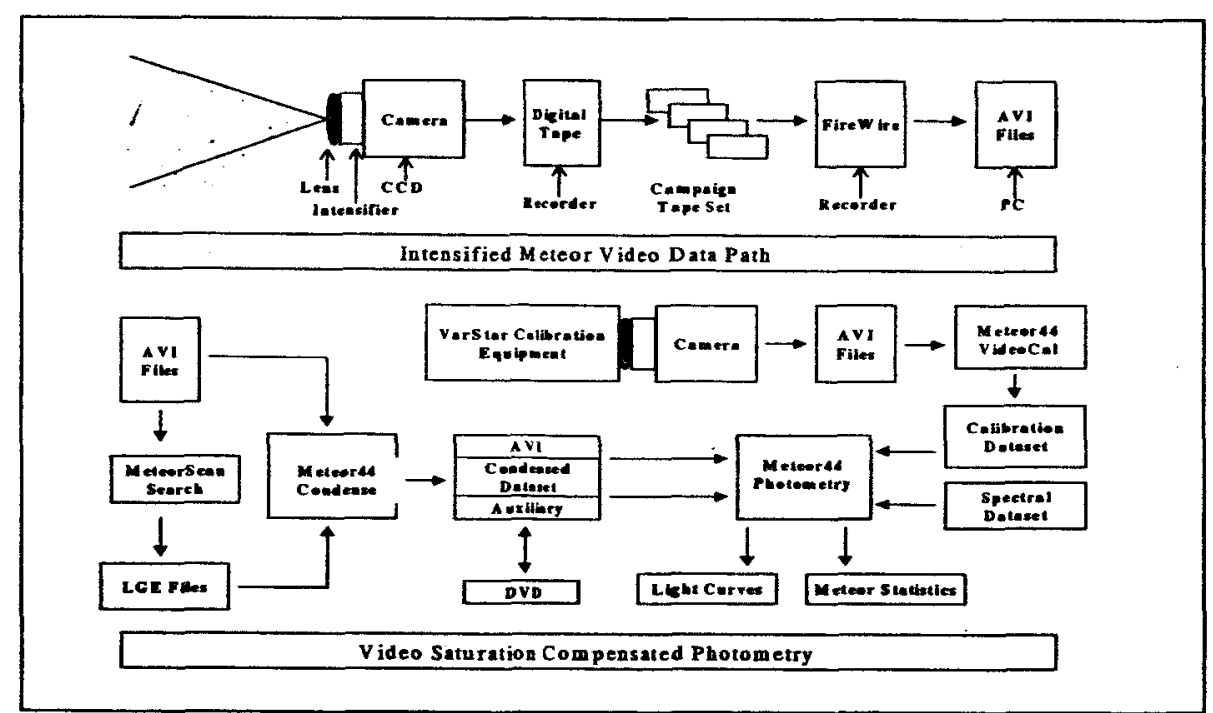

Figure 1 a) Intensified metcor video data path from the sky to the PC. b) Data flow for saturation compensated video photometry with artificial variable star (VarStar) calibration.

\subsection{Meteor44 Sky glow Derived Flatfield and Background}

Meteor44 automatically derives a flatfield from the sky glow, which is assumed flat. This is a reasonable assumption for moderate fields of view (FOV) more than 20 degrees above the horizon after astronomical twilight. Most observations are made under these conditions and a single flatfield can be used for a complete night's observations. The procedure averages several frames of meteor-free sky data, removes the stars and small-scale variations by median filtering and smooths the result. This result is normalized to unity in the center, bounded and inverted to yield a normalized, multiplying flatfield. In most cases, the flatfield produced is consistent with the classic $1 / \cos ^{4} \Theta$ curve of a widefield lens [Kingslake, 1989]. Note that the compensation in the useful portion near the edge of the image approaches a factor of 2 or visual magnitude, $\Delta \mathrm{m}_{\mathrm{y}}=0.75$.

A related method is used to subtract the airglow background from the data. The stars and meteors are removed from an image as above and this background is subtracted. In practice, radial aperture photometry, described below, reduces the need for whole-image background subtraction since it accounts for changes in local background conditions. In Meteor44, the subtraction of the sky background is used primarily to improve the performance of automated object location algorithms.

\subsection{Meteor44 Stellar Photometry and Astrometry}


Radial aperture photometry (RAP) is used in Meteor44. One determines the mean intensity in each ring about the centroid of the target object, and from this finds the radial point spread function (PSF). From the radial PSF, appropriate target and background radii are determined then target and background intensities are calculated as with traditional circular aperture photometry. The local background is determined using a robust Mean-Median-Half technique to avoid artifacts in crowded regions. Sensitivity to nearby stars is greatly reduced with the technique as the target aperture is no larger than necessary and robust local backgrounds are used. The technique has been adapted to meteor intensity measurements by adding an algorithm to estimate the meteor trail length to width ratio as in section 7.0.

The star catalog used by Meteor 44 is the Sky2000 catalog, [Goddard, 2000] compiled for attitude determination star trackers. This catalog is an improvement over earlier general catalogs in that the $m_{B}$ and $m_{V}$ magnitudes are far more complete and accurate, the astrometry has been updated and selected $m_{R}$ and $m_{1}$ magnitudes are available as well. This is important since the GENIII detectors often chosen for meteor work are very sensitive in the near infrared. Color information from stars in the Landolt selected area catalog [Landolt, 1992] was used to perform a statistical regression relating V-R and V-I to B-V in order to estimate missing red and infrared magnitudes as suggested by Holtzman [2001].

To initialize the astrometry, the user clicks on several corresponding stars in both the sky image and a catalog image so a plate solution can be computed. Using the plate solution and catalog, Meteor44 automatically locates a dozen or more stars, applies RAP and determines their instrument response. Much of the automated astrometry in Meteor44 is adapted from previous instrument pointing programs [Dietz, 2002] developed for NASA. Spectral and saturation compensation are used as discussed in sections 5.0 and 6.0 below to refine the instrument calibrations.

\subsection{Stellar \& Meteoric Spectral Response}

Photometric calibration from the stars in the video images is wavelength sensitive. For simplicity, the process is broken into two parts by the determination of the instrument magnitude, $\mathbf{M}_{\mathrm{G}}$, based upon the photon response of the instrument. One convolves a standard spectrum [Colina et al. 1996] of the standard star Vega, Figure $2 \mathrm{a}$, and the quantum efficiency $(\mathrm{QE})$ spectrum of the video detector, Figure $2 b$, to get $M_{G}=0$ instrument response in photon units. The magnitude and color information of catalog stars in the field of view is used to estimate their spectra, which is compared to the measured instrument photon response to get a calibration in terms of instrument units and the stellar saturation threshold, Sat. The meteor instrument response in each field is converted into $\mathrm{M}_{\mathrm{G}}$, and these are combined to form the meteor light curve. Compensation (sections 6.0 and 7.0) is applied as required.

To compare $\mathbf{M}_{\mathrm{G}}$ photometric results to visual observations or to compare with results from another instrument requires conversion to equivalent visual magnitude, $M_{V}$. Establishing the relationship between meteoric $M_{G}$ and $M_{V}$ requires knowledge of the meteor spectrum, Figure $2 b$, the instrument quantum efficiency curve and the V-filter [Landolt, 1992] spectrum, Figure 2a. The ratio of the meteor spectrum convolved with the $\mathrm{QE}$ to the meteor spectrum convolved with the V-filter curve is used to estimate the $\mathrm{M}_{\mathrm{G}}$ to $\mathrm{M}_{\mathrm{V}}$ offset. This offset added to the meteor light 
curve in $\mathbf{M}_{\mathrm{G}}$ yields the light curve in $\mathrm{M}_{\mathrm{V}}$ and the peak value for comparison to visual estimates.

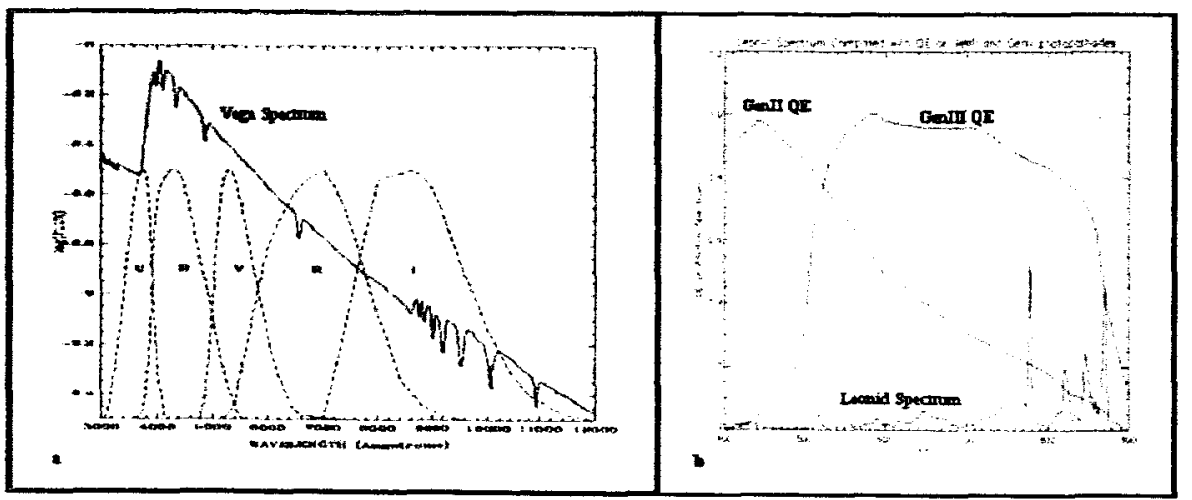

Figure 2 a) The Vega spectrum compared with the bandpass of Landolt UBVRI astronomical fiters. b) The quantum efficiency (QE) spectrum of GENII and GENIII photocathodes compared with a sample Leonid spectrum. The response to the bright IR lines is quite different.

\subsection{Saturation Compensation from "Artificial Variable Star"}

The theory and construction of "artificial variable star" video calibration hardware is fairly straightforward. Light through a motorized ND3 neutral density filter varying in intensity by 1000:1 over a two second period is focused upon a pinhole. Light from the pinhole is collimated by a telescope and is observed by the camera under test as a variable star at infinity (varstar). Neutral density filters are used to adjust the intensity so that the varstar image ranges from dim to extremely saturated. Five or ten cycles of data are recorded and processed in the usual manner.

The varstar in each field of the data is measured using RAP and correlated with filter wheel position. The power law coefficients that fit the saturated data to the filter wheel curve comprise the saturation calibration for this camera-lens combination. Although other saturation calibration function fits may be used, the best results are usually found using geometric (power law) fitting of the data to the wheel. For poorly focused systems the saturation calibration exponent can approach unity (i.e. linear) but the usual range of the calibration exponent for intensified cameras with sharp lenses is from 1.2 to 1.5. The slope of the compensation is used to estimate errors.

\subsection{Motion Compensation: Comparing Stellar and Meteoric Images}

Motion smearing spreads the intensity thinner than for a stellar source. This has a significant effect on the saturation threshold and the saturation compensation for the meteor. One can analyze a smeared meteor track as a prismatic mid portion (translation of the PSF) with a half circular portion on each end, as shown in Figure 3. The central white portion represents the saturated region. The PSF and the length to width ratio, $L W$, are sufficient to describe the figure produced. For a stellar image, there is no prismatic portion and the ratio is unity. For a meteor, the ratio depends on PSF width and the movement of the meteor 
during the exposure. For extremely bright meteors the PSF is broad and the ratio approaches unity.

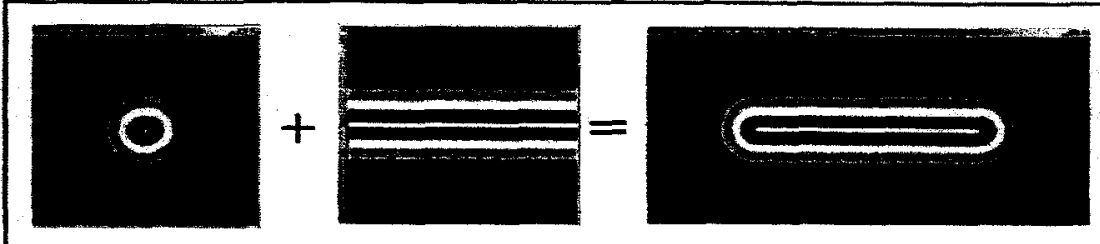

Figure 3, Geometrically, a short meteor exposure can be seen as the combination of a circular (stellar) component on each end and a linear, prismatic component in the center. Imagine a prism and a cone.

$L$ and $W$ are found from the saturated spot by examining the spot's radial intensity function within the RAP procedure: all pixels are saturated up to radius $W$ and no pixels are saturated beyond radius $L$. If one defines the stellar saturation threshold, Sat, as the minimum total intensity which will produce four saturated pixels, then one can scale this threshold for a star to that of meteor, $S a t_{m}$ as a function of $L W$ based on the ratio of geometric areas, $A_{\text {meteo }} / A_{\text {spot }}$.

The final meteor saturation compensation step is to apply the power law saturation technique from section 6.0 for stellar images replacing Sat with $\mathrm{Sat}_{m}$ as the saturation level. Below an integrated meteor intensity of Sat $_{m}$ meteor saturation compensation is not required. Above an integrated meteor intensity of $\mathrm{Sat}_{m}$, the camera saturation compensation is determined by artificial variable star methods.

\subsection{Application to the Bright 2001 Leonids}

The 2001 Leonid meteor storm over North America was characterized by an extraordinary number of very bright meteors with fewer dim meteors than expected. Analysis of the intensified video data from this meteor storm provided both the motivation and the prototype for the development of these procedures. Almost all meteors were very saturated (Figure 4) and there would have been little usable data if the saturated meteors had been tossed out. Deviations from the line are due to light curve shape effects: flat light curves have peak $m_{G}$ values below the line, while spiked light curves will have peak $\mathrm{m}_{\mathrm{G}}$ values above the line in Figure 4.

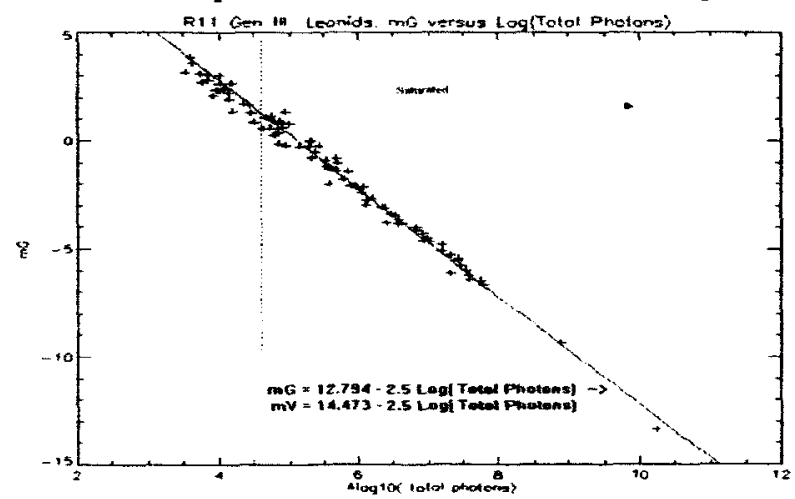

Figure 4, Meteors recorded with intensified video over Hawaii November, 2001. Note the dynamic range (instrument magnitude $M_{G}=4$ to -13 ) and numerous extremely bright meteors. Here $M_{V}=M_{G}+1.68$ 


\section{Acknowledgements}

The authors wish to acknowledge Peter Gural for making modifications to MeteorScan to read and process AVI files and Peter Brown of the University of Western Ontario for the loan of several GENIII and GENII intensified cameras and associated equipment for use in our observing campaigns. Mr. Swift was supported with funding from NASA contract NAS8-00187

\section{Bibliography}

Borovicka, H., Stork, R., Bocek, J., 1999, "First results from video spectroscopy of 1998 Leonid meteors", Meteoroitics \& Planet. Sci. 42 145-150

Colina, L., Bohlin, R.., Castelli, F. 1996, “Absolute Flux Calibrated Spectrum of Vega". Instrument Science Report CALSCS-008

Dietz, K. L., Ramsey, B. D., Alexander, C. D., Apple, J. A., Ghosh, K. K.\& Swift, W. 2002, "A Daytime Aspect Camera for Balloon Altitudes", Opt. Eng., 41, n. 10 2641

Goddard, SKY2000 Master Catalog, Version 3 (Rev. 1), Goddard Space Flight Center, Flight Dynamics Division, 2000

Gural, P. 1995 “An operational autonomous meteor detector: Development issues and early results". WGN: J. Inter. Meteor Org. 25, 136-140.

Holtzman, J., private communication, New Mexico University, 2001

Kingslake, R. 1989 A History of the Photographic Lens, Academic Press, San Diego.

Landolt, A.U.,1992, "UBVRI Photometric Standard Stars in the Magnitude 